\title{
A Concise Synthesis of a Methyl Ester 2-Resorcinarene: A chair-conformation macrocycle
}

\author{
Michael R. Reynolds, Fraser S. Pick, John J. Hayward* \& John F. Trant* \\ Department of Chemistry and Biochemistry, University of Windsor, 401 Sunset Ave., ON N9B \\ 3P4 Windsor, Canada; jhayward@uwindsor.ca; j.trant@uwindsor.ca
}

\begin{abstract}
Anions are important hydrogen bond acceptors in a range of biological, chemical, environmental and medical molecular recognition processes. These interactions have been exploited for the design and synthesis of ditopic resorcinarenes as the hydrogen bond strength can be tuned through the modification of the substituent at the 2-position. However, many potentially useful compounds, especially those incorporating electron-withdrawing functionalities, have not been prepared due to the challenge of their synthesis: their incorporation slows resorcinarene formation that is accessed by electrophic aromatic substitution. As part of our broader campaign to employ resorcinarenes as selective recognition elements, we need access to these specialized materials, and in this article we report a straightforward synthetic pathway for obtaining a 2(carboxymethyl)-resorcinarene, and resorcinarene esters in general. We discuss the unusual conformation it adopts, and propose that this arises from the electron-withdrawing nature of the ester substituents that renders them better hydrogen bond acceptors than the phenols, ensuring that each of those acts as a donor only. DFT calculations show that this conformation arises as a consequence of the unusual configurational isomerism of this compound and interruption of the archetypal hydrogen bonding by the ester functionality.
\end{abstract}

Keywords: Supramolecular chemistry; anion-mediated hydrogen bonds; density functional theory; polymorph. 


\section{Introduction}

Resorcinarenes are (usually)-bowl-shaped macrocyclic compounds stabilised by a circular network of intramolecular $\mathrm{O} \cdots \mathrm{H}-\mathrm{O}$ hydrogen bonds.[1, 2] These compounds represent a unique family of host compounds which have been extensively studied in supramolecular host-guest chemistry because they display several sites for non-covalent interactions, excellent $\mathrm{p} K_{\mathrm{a}}$ tunability, and an electron rich bowl-shaped cavity in the $C_{4 \mathrm{v}}$ symmetric conformation, among a myriad of other interesting properties.[2-4] Their cavity can accommodate a wide range of guest molecules through non-covalent interactions including (but not limited to) hydrogen bonding, halogen bonding, cation $\cdots \pi, \mathrm{C}-\mathrm{H} \cdots \pi$ as well as $\pi \cdots \pi$ interactions depending on both the size and charge distribution of the respective guest molecules and the functionalization of the resorcinarene. ${ }^{4}$ In addition to their structural role enforcing the upper rim of the macrocycle, the hydroxyl groups at the 1 and 3 positions on the aromatic subunits can participate extensively in hydrogen bonding with hydrogen bond accepting guest molecules.[5-9] As a direct result of these hydrogen bonded supramolecular networks, resorcinarenes have been extensively exploited as appropriate hosts to accommodate a myriad of guests ranging from alcohols,[10-14] to sugars,[15-18] steroids[19-21] and even heterocyclic five- and six-membered ring compounds as guest molecules.[22-26]

On resorcinarenes themselves, reaction at $\mathrm{C} 2$ is selective over $\mathrm{C} 4$ and $\mathrm{C} 6$ positions, as these are blocked by the lower rim linkages of the resorcinarene ring. The hydrogen bonded network of hydroxyl groups enhances the acidity of the phenol while increasing $\pi$-basicity inside the cavity.[27] Attenuation or cleavage of the $\mathrm{O}-\mathrm{H}$ bonds, exo to the upper rim, by bases results in increased electron density on the oxygen, effectively strengthening the hydrogen bonding.[26, 2830] 


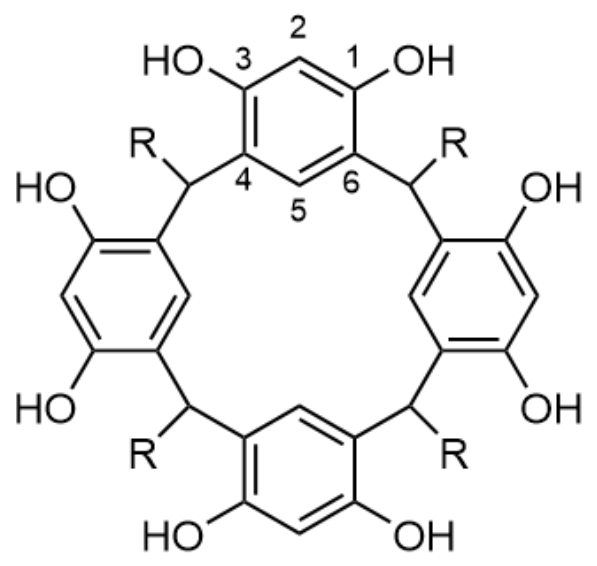

Figure 1: A generic resorcinarene illustrating the numbering convention

Functionalization of resorcinarenes at the 2-position tunes the relative acidity of the phenolic hydrogens allowing for selective reactions with certain guests. Deprotonation of the phenolic hydrogens with amine bases creates protonated ammonium cations which form interesting supramolecular complexes with the anionic resorcinarenes. These assemblies may have enhanced crystallinity that can then be studied both in the solid state and solution state by single crystal X-ray diffraction and ${ }^{1} \mathrm{H}$ NMR respectively, as well as in the gas phase by mass spectrometry. The challenge is to access a wide enough variety of resorcinarenes to take advantage of these potential specific interactions. As part of our campaign to access a greater variety of these molecules, we wish to report the synthesis of a simple ester resorcinarene, and its very unresorcinarene like conformation.

\section{Results and Discussion}

A resorcin[4]arene with an ester functionality in the 2-position has not been reported; this moiety would act as an electron-withdrawing functionality that would increase the acidity of the phenols. 
The formation of resorcinarene macrocycles as crystalline solids with high melting points through the acid-catalyzed condensation of resorcinol (or functionalized resorcinols) with aldehydes is well established.[1] Högberg was one of the first to discover the synthesis of resorcinarenes using formaldehyde and resorcinol in acidic conditions.[31] This approach works extremely well for simple 2-haloresorcinarenes and we have found success employing it for other functionalities, so it was the starting point for our synthesis.[32]

To obtain 2-substituted resorcinarenes functionalization can take place either before or after cyclisation. As macrocycle formation blocks the 4 and 6 positions the post-cyclisation strategy can have advantages in terms of regioselectivity, although as four functional group transformations must occur in every step, incomplete substitution can lead to complex mixtures, difficult purification and low yields. Pre-cyclization methods, by contrast, introduces regioselectivity issues, but the use of a purified monomer ensures uniform substitution in the macrocycle. In this case we pursued a pre-cyclisation derivatization protocol because of the ready availability of a suitable precursor; the monomer unit was readily obtainable via a slow Fischer esterification of commercially available 2,6-dihydroxybenzoic acid using sulfuric acid in methanol. Following removal of the solvent in vacuo, the residue was dissolved in dichloromethane and washed with saturated sodium bicarbonate, which removed any unreacted starting material along with the sulfuric acid catalyst. Pure methyl 2,6-dihydroxy benzoate was obtained as a pinkish solid in 51\% yield (Scheme 1). 


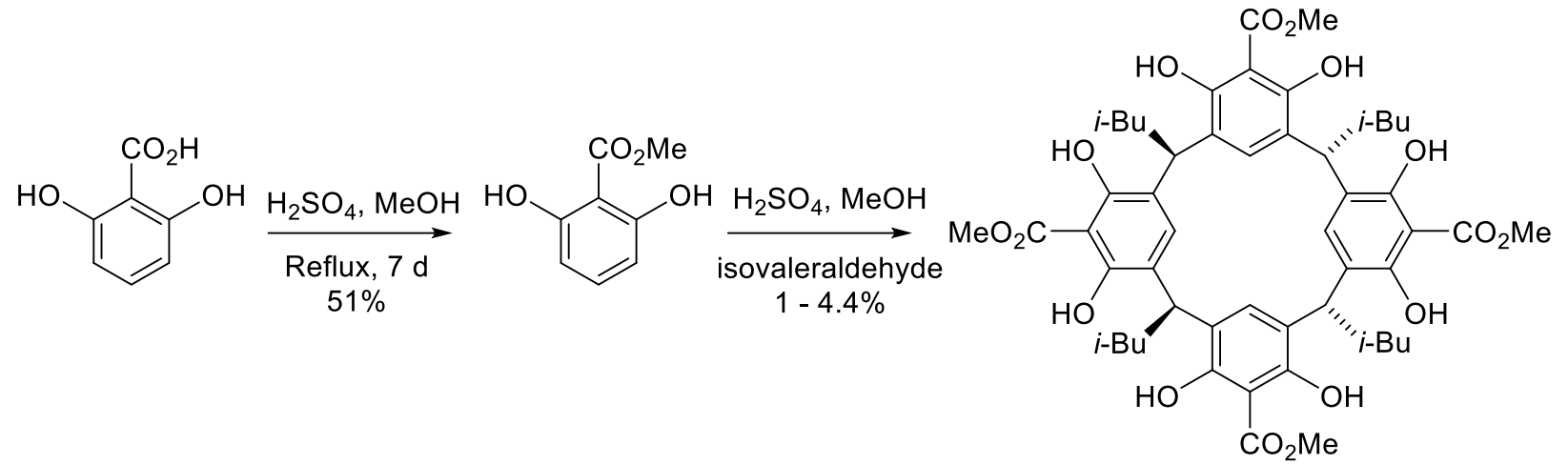

Scheme 1: Synthesis of the resorcin[4]arene from 2,6-dihydroxybenzoic acid.

With the functionalized resorcinol in hand, several approaches toward macrocyclization were attempted using isovaleraldehyde, as the tetra isobutyl resorcinarenes are typically highly crystalline in our experience. Initially, an acid catalyzed cyclization using the methodology from Högberg,[31] a 2:2:1 v/v mixture of methanol, water and concentrated hydrochloric acid at reflux over several days, was attempted. However, upon workup a complex mixture was observed that included partial hydrolysis of the methyl ester moieties, and a significant amount of acyclic oligomeric and polymeric material, presumably as a consequence of the high water content in the mixture; none of the tetra ester product could be detected. Attempts to re-esterify this complex mixture resulted in decomposition of the material. This suggests that it might be difficult to access the ester from the known 2-carboxy resorcinarene.[33]

As an alternative, we know that the electron-withdrawing ester group decreases the $\mathrm{p} K_{\mathrm{a}}$ of the phenol groups relative to the unsubstituted homologue making them easy to deprotonate; the resulting phenolates would increase the nucleophilicity of the resorcinol, and any undesired oxygen-centered nucleophilic attack on the aldehyde electrophiles would be non-productive. Consequently, we attempted a base-catalyzed approach. Bourgeois has effected the 
macrocyclization of 2-nitroresorcin[4]arene using sodium hydroxide in water;[34] instead we used a solution of sodium methoxide in methanol so as not to hydrolyze the methyl ester. Upon quenching with acid and filtration, a complex mixture was observed that showed ${ }^{1} \mathrm{H}$ NMR signals at approximately $6.3 \mathrm{ppm}$. This suggested that the aldehyde component had undergone an aldol self-condensation as the major reaction. It should be noted that any of the resorcinarenes reported to date that were cyclized under basic conditions were only done so using formaldehyde as the aldehyde component. Lacking any $\alpha$-protons, enolate formation (and hence aldol reaction) is impossible in these cases, explaining why this complication hasn't been reported, though it has likely been encountered. However, no hydrolysis of the ester was observed. Combining these insights, we were able to effect the desired macrocyclization by employing concentrated sulfuric acid in methanol, providing the desired resorcinarene as a white solid in a poor 1-4.4\% yield over repeated trials, with a great majority of the lost mass balance attributed to the formation of polymer and oligomer (Scheme 1). Curiously the NMR was not as we expected and gave us grave concern (Figure 2). Generally, resorcinarenes are, as we have emphasized, found in a $C_{4 \mathrm{v}}$ symmetric bowlshaped conformation. In this form, the protons on each of the subunits is magnetically equivalent with its congeners on the others. Consequently, one only observes a single aromatic signal, a single benzylic signal, and a single set of peaks for the lower rim alkyl chain. This is not what we found. Instead, our spectrum was consistent with a pair of isomers. We spent a significant amount of time examining this challenge but the apparent mixture behaves as a single compound on TLC, and HPLC, and we could never separate these signals. Seeking clarification on this issue, we attempted to recrystallize, but this also did not change the ratio of the signals or enrich our sample in either compound. However, it did provide us with material of sufficient quality for X-Ray analysis. 

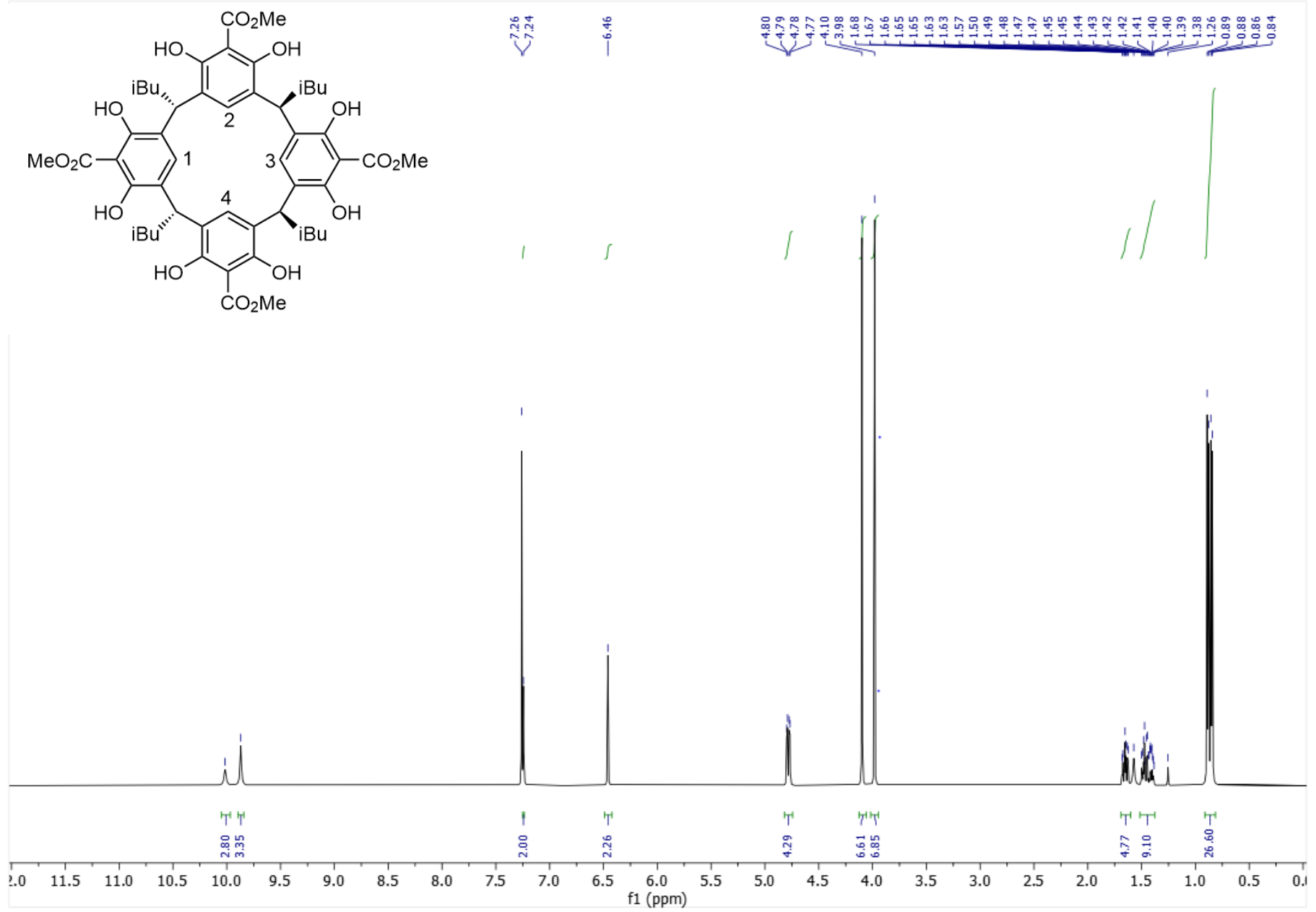

Figure 2: ${ }^{1} \mathrm{H}$ NMR spectrum showing $C_{2}$ configuration in solution

Crystals suitable for single-crystal X-ray diffraction could be obtained from the white powder by slow evaporation of a chloroform solution (Figure 3). The crystal structure of the obtained compound revealed two unusual features. Firstly, the configuration of the isobutyl groups around the lower rim of the resorcinarene is reminiscent of $C_{2}$ symmetry (this can be seen in the 2D representation in Figure 4a), in contrast to the more commonly observed $C_{4 v}$ isomer. Secondly, in the majority of resorcinarene crystal structures, the observed conformation is the archetypal bowl 
shape. This crystal instead exhibited a "chair" conformer with a pseudo- $C_{2}$ rotation axis where two of the resorcinol subunits ( 2 and 4, see Figure 2 inset for numbering) are coplanar with one another while the other two (1 and 3) sit orthogonal to the plane and antiperiplanar to one another. This result also clearly contextualizes the doubling of the resonances in the NMR spectra: this conformation is not an artifact of crystallization but appears to persist in solution and not rapidly interconvert or "flip" the pseudochair, in which case we would observe a single set of peaks as the average of the two chemical environments. The NMR spectrum can now be understood in terms of this conformational preference, where the two $6 \mathrm{H}$ singlets at 4.10 and $3.98 \mathrm{ppm}$ correspond to the methyl esters in two different environments. This is consistent with the pseudochair conformation, where rings $1 \& 3$ are related by a $C_{2}$ rotation axis (through the $\mathrm{C} 2 / \mathrm{C} 5$ atoms of rings $2 \& 4)$ and $2 \& 4$ are related by a mirror plane that bisects rings $1 \& 3$. We are currently developing a model to explain this unusual conformation, and stereoisomeric product, and are also preparing additional electron-poor members that might show similar behaviour. However, we speculate that without the phenols working together to form the hydrogen bond network and template the forming resorcinarene, the typical $C_{4 v}$ conformation might not be favoured.
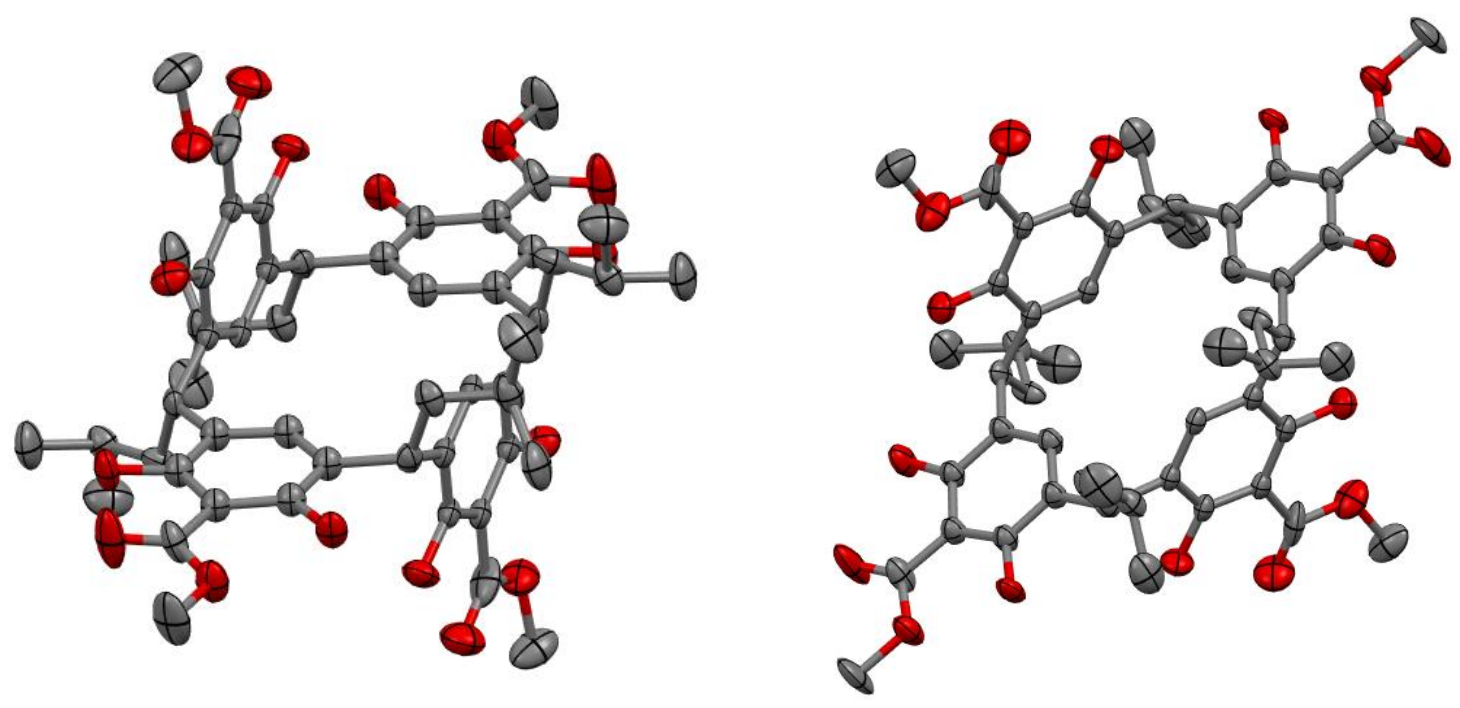
Figure 3. Front and side view of the crystal structure of 2-(methylcarboxyl)resorcin[4]arene with elipsoids drawn at 50\% probability.. H-atoms omitted for clarity. Deposition \#2070167 in the CCDC.

To investigate the unusual conformational preference of the resorcinarenes, DFT calculations were performed at the $\omega \mathrm{B} 97 \mathrm{XD} / 6-311 \mathrm{G}(\mathrm{d}, \mathrm{p})$ level of theory in the gas phase and using the polarized continuum solvation model (PCM) to consider solvent effects. Geometric optimizations of the "chair" conformation and a theoretical "bowl" geometry were performed. The initial geometry for the "chair" conformer was obtained from the solid-state molecular structure, whereas the "bowl" conformer was based on solid state molecular structure of known resorcinarenes. The energies and structures of the solvent-corrected optimized conformations are provided in Figure 4a; the optimised structures (as .mol2 files), gas phase energies and all thermodynamic parameters can be found as Supporting Information. These calculations showed that the classic resorcinarene bowl conformation was disfavoured by $20.5 \mathrm{kcal} / \mathrm{mol}$ using the solvent correction $(25.7 \mathrm{kcal} / \mathrm{mol}$ in the gas phase $)$, an enormous preference for the observed conformer. This large preference has two possible contributing factors: first, the ester functional groups are Lewis basic and therefore have the ability to act as hydrogen-bond acceptors to the phenolic hydrogen bond donors when coplanar to the benzene ring. Second, most resorcinarenes are found as the $C_{4 \mathrm{v}}$ configurational isomer; the steric hinderance of the isobutyl groups in the isomer obtained in this case may also impact the conformational preference of the macrocycle. 
a) $C_{2}$ configuration

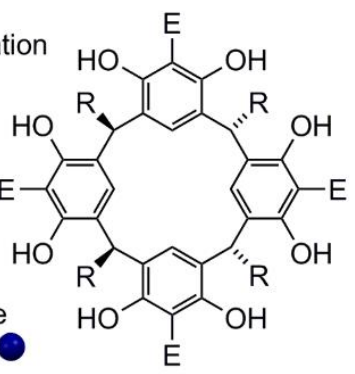

b) $C_{4 v}$ configuration

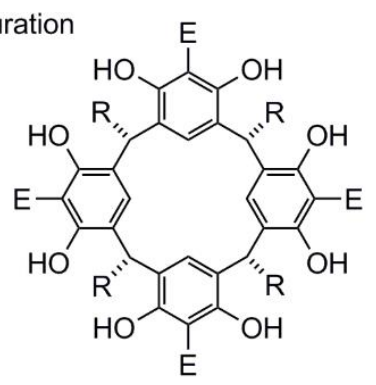

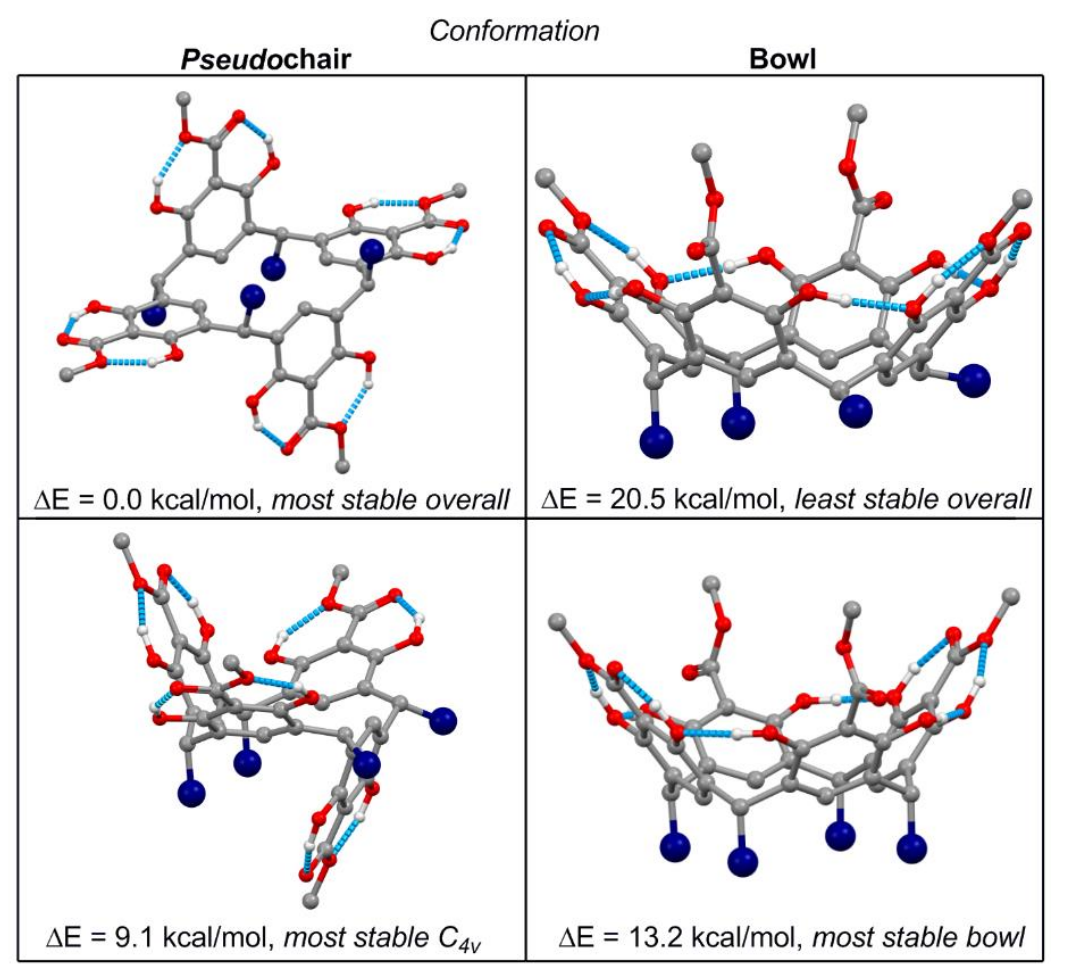

Figure 4: Computed energies of a) the obtained $C_{2}$ isomer and b) expected $C_{4 \mathrm{v}}$ isomer. All $\mathrm{C}-\mathrm{H}$ bonds have been omitted and the isobutyl groups are represented by navy spheres for clarity. Hydrogen bonds have been highlighted in blue. Note: the configuration refers purely to the relative arrangement of the isobutyl groups.

To investigate the impact of these factors, chair and bowl structures of a $C_{4 \mathrm{v}}$ resorcinarene were also calculated (Figure 4b). The chair conformer is still preferred in these structures, although the preference is much reduced compared to the $C_{2}$ isomer; $(4.1 \mathrm{kcal} / \mathrm{mol}$ using the chloroform solvent correction, $9.8 \mathrm{kcal} / \mathrm{mol}$ in the gas phase). This shows that the configuration at the carbons bridging the resorcinol subunits can have a significant effect on the conformational preference of the macrocycle, but is not the most important factor in this case. The upper rim of a resorcinarene bowl is formed by a hydrogen bond network; this is interrupted by the presence of the esters as hydrogen bond acceptors. The esters in the crystal structure are all coplanar with the 
benzene rings; this maximises the delocalisation of electron density from the electron-rich ring into the carbonyl of the ester, which enhances its Lewis basicity. This likely has a synergistic effect with the hydrogen bond-donor phenols, which will hold the ester coplanar. It should be noted that delocalisation is not the only reason for coplanarity; in each of the computed bowl structures two of the ester groups rotate out of the plane in the absence of this hydrogen bonding. We can therefore conclude that the ester acting as a hydrogen bond acceptor therefore has the most significant effect upon the conformational preference of this macrocycle. Investigations into the generality of this phenomenon are underway in our laboratory.

\section{Conclusion:}

We have successfully synthesized a novel 2-methyl ester resorcin[4]arene under simple acid catalyzed conditions, if in poor yield, and the structure and solid-state conformation were determined by single crystal X-ray diffraction and NMR spectra. Computational investigations of this system revealed a significant preference for the observed pseudochair conformation and shed light upon the interplay of configurational and hydrogen bonding effects that are in operation in resorcinarene structures. Our studies in this area will further investigate the conformational preference of this and related systems, and methods to rationalise and control this aspect of supramolecular architecture will be developed.

Conflicts of Interest: The authors claim no conflicts of interest.

Supporting Materials: The structure of 2-(methylcarboxyl)resorcin[4]arene has been deposited in the Cambridge Crystal Databank (\#2070167). The geometry-optimized structures (as .mol2 files), video surveys of the crystal and calculated structures, including a superimposition of the 
crystal structure with the minimum energy calculated $C_{2}$ pseudochair configuration, complete methods and materials are found in the accompanying supporting information.

CREDIT: Conceptualization, JFT; Funding acquisition, JFT; Investigation, MRR, JJH; Methodology, MRR, JJH, FP; Visualization and crystal analysis, FP; Project administration, JFT; Supervision, JFT, JJH; Writing original draft, MRR; Writing-review and editing, all authors.

Acknowledgements: The authors would like to thank the Natural Sciences and Engineering Research Council of Canada Discovery Grants Program (NSERC, 2018-06338 to JFT) the

Canadian Foundation for Innovation and Ontario Research Fund (37425), and MITACS and Odd Society Spirits (IT13760, salary to FP) for support for this work. The authors would like to thank

Lara Watanabe, University of Windsor, for acquiring the crystallographic data.

\section{References:}

1. Sliwa, W.; Kozlowski, C., Calixarenes and Resorcinarenes. Wiley: Weinheim, Germany, 2009.

2. Timmerman, P.; Verboom, W.; Reinhoudt, D. N., Resorcinarenes. Tetrahedron 1996, 52, (8), 2663-2704.

3. Böhmer, V., Calixarenes, Macrocycles with (Almost) Unlimited Possibilities. Angewandte Chemie International Edition in English 1995, 34, (7), 713-745.

4. Rissanen, K., Very Large Container Molecules. Angewandte Chemie International Edition 2005, 44, (24), 3652-3654.

5. Atwood, J. L.; Szumna, A., Anion-sealed single-molecule capsules. Chemical Communications 2003, (8), 940-941.

6. Atwood, J. L.; Barbour, L. J.; Jerga, A., Organization of the interior of molecular capsules by hydrogen bonding. Proceedings of the National Academy of Sciences 2002, 99, (8), 4837-4841.

7. R. MacGillivray, L.; L. Atwood, J., Unique guest inclusion within multi-component, extendedcavity resorcin[4]arenes. Chemical Communications 1999, (2), 181-182.

8. Beyeh, N. K.; Kogej, M.; Åhman, A.; Rissanen, K.; Schalley, C. A., Flying Capsules: Mass Spectrometric Detection of Pyrogallarene and Resorcinarene Hexamers. Angewandte Chemie International Edition 2006, 45, (31), 5214-5218.

9. Shivanyuk, A.; Paulus, E. F.; Rissanen, K.; Kolehmainen, E.; Böhmer, V., Resorcarenes in the Boat Conformation as Building Blocks for Hydrogen-Bonded Assemblies Including Two Ammonium Cations. Chemistry - A European Journal 2001, 7, (9), 1944-1951.

10. Beyeh, N. K.; Weimann, D. P.; Kaufmann, L.; Schalley, C. A.; Rissanen, K., lon-Pair Recognition of Tetramethylammonium Salts by Halogenated Resorcinarenes. Chemistry - A European Journal 2012, 18, (18), 5552-5557. 
11. Avram, L.; Cohen, Y.; Rebek Jr, J., Recent advances in hydrogen-bonded hexameric encapsulation complexes. Chemical Communications 2011, 47, (19), 5368-5375.

12. Koshets, I. A.; Kazantseva, Z. I.; Belyaev, A. E.; Kalchenko, V. I., Sensitivity of resorcinarene films towards aliphatic alcohols. Sensors and Actuators B: Chemical 2009, 140, (1), 104-108.

13. Ugono, O.; Holman, K. T., An achiral form of the hexameric resorcin[4]arene capsule sustained by hydrogen bonding with alcohols. Chemical Communications 2006, (20), 2144-2146.

14. Fox, O. D.; Leung, J. F. Y.; Hunter, J. M.; Dalley, N. K.; Harrison, R. G., Metal-Assembled Cobalt(II) Resorc[4]arene-Based Cage Molecules That Reversibly Capture Organic Molecules from Water and Act as NMR Shift Reagents. Inorganic Chemistry 2000, 39, (4), 783-790.

15. Kalenius, E.; Kekäläinen, T.; Neitola, R.; Beyeh, K.; Rissanen, K.; Vainiotalo, P., Size- and Structure-Selective Noncovalent Recognition of Saccharides by Tetraethyl and Tetraphenyl Resorcinarenes in the Gas Phase. Chemistry - A European Journal 2008, 14, (17), 5220-5228.

16. He, M.; Johnson, R. J.; Escobedo, J. O.; Beck, P. A.; Kim, K. K.; St. Luce, N. N.; Davis, C. J.; Lewis, P. T.; Fronczek, F. R.; Melancon, B. J.; Mrse, A. A.; Treleaven, W. D.; Strongin, R. M., Chromophore Formation in Resorcinarene Solutions and the Visual Detection of Mono- and Oligosaccharides. Journal of the American Chemical Society 2002, 124, (18), 5000-5009.

17. Evan-Salem, T.; Baruch, I.; Avram, L.; Cohen, Y.; Palmer, L. C.; Rebek, J., Resorcinarenes are hexameric capsules in solution. Proceedings of the National Academy of Sciences 2006, 103, (33), 12296.

18. Rhlalou, T.; Ferhat, M.; Frouji, M. A.; Langevin, D.; Métayer, M.; Verchère, J. F., Facilitated transport of sugars by a resorcinarene through a supported liquid membrane. Journal of Membrane Science 2000, 168, (1), 63-73.

19. Shivanyuk, A.; Rebek, J. J., Hydrogen-bonded capsules in polar, protic solvents. Chemical Communications 2001, (22), 2374-2375.

20. Faull, J. D.; Gupta, V. K., Chemical selectivity of self-assembled monolayers of calix[4]resorcinarene. Thin Solid Films 2003, 440, (1), 129-137.

21. Faull, J. D.; Gupta, V. K., Impact of Host Structure on Guest-Host Recognition at Self-Assembled Surfaces of Tetrathiol and Tetrasulfide Derivatives of Calix[4]resorcinarene. Langmuir 2002, 18, (17), 6584-6592.

22. Puttreddy, R.; Beyeh, N. K.; Jurček, P.; Turunen, L.; Trant, J. F.; Ras, R. H. A.; Rissanen, K., Hostguest complexes of C-propyl-2-bromoresorcinarene with aromatic $\mathrm{N}$-oxides. Supramol. Chem. 2018, 30, (5-6), 445-454.

23. Puttreddy, R.; Beyeh, N. K.; Taimoory, S. M.; Meister, D.; Trant, J. F.; Rissanen, K., Host-guest complexes of conformationally flexible $\mathrm{C}$-hexyl-2-bromoresorcinarene and aromatic $\mathrm{N}$-oxides: solid-state, solution and computational studies. Beilstein J. Org. Chem. 2018, 14, 1723-1733.

24. Puttreddy, R.; Beyeh, N. K.; Ras, R. H. A.; Trant, J.; Rissanen, K., Endo-/exo- and halogen bonded complexes of conformationally rigid $\mathrm{C}$-ethyl-2-bromoresorcinarene and aromatic $\mathrm{N}$-oxides. CrystEngComm 2017, 19, 4312-4320.

25. Nissinen, M.; Rissanen, K., Crystal Engineering Studies of the Complexes of Ethyl Resorcinarene with Aromatic Nitrogen Heterocycles. Supramolecular Chemistry 2003, 15, (7-8), 581-590.

26. Nissinen, M.; Wegelius, E.; Falábu, D.; Rissanen, K., Melamine induced conformational change of ethyl resorcinarene in solid state. CrystEngComm 2000, 2, (28), 151-153.

27. Taimoory, S. M.; Twum, K.; Dashti, M.; Pan, F.; Lahtinen, M.; Rissanen, K.; Puttreddy, R.; Trant, J. F.; Beyeh, N. K., Bringing a molecular plus one: Synergistic binding creates guest-mediated threecomponent complexes. J. Org. Chem. 2020, 85, (9), 5884-5894.

28. Zheng, S.-L.; Gembicky, M.; Messerschmidt, M.; Dominiak, P. M.; Coppens, P., Effect of the Environment on Molecular Properties: Synthesis, Structure, and Photoluminescence of $\mathrm{Cu}(\mathrm{I})$ 
Bis(2,9-dimethyl-1,10-phenanthroline) Nanoclusters in Eight Different Supramolecular Frameworks. Inorganic Chemistry 2006, 45, (23), 9281-9289.

29. Murayama, K., Resorcin[4]arene dimer linked by eight water molecules and incorporating a tetraethylammonium ion: guest-driven capsule formation via cation $-\pi$ interactions. Chemical Communications 1998, (5), 607-608.

30. Atwood, J. L.; Barbour, L. J.; Hardie, M. J.; Lygris, E.; Raston, C. L.; Webb, H. R., Inclusion complexes of 18-crown- 6 and ( $\mathrm{Na}+\subset$ [2.2.2]cryptand) in [C-methylcalix[4]resorcinarene-Hn], $\mathrm{n} \bigoplus=\bigoplus 0,1$. CrystEngComm 2001, 3, (10), 41-43.

31. Högberg, A. G. S., Two stereoisomeric macrocyclic resorcinol-acetaldehyde condensation products. The Journal of Organic Chemistry 1980, 45, (22), 4498-4500.

32. Beyeh, N. K.; Pan, F.; Rissanen, K., A Halogen-Bonded Dimeric Resorcinarene Capsule. Angewandte Chemie International Edition 2015, 54, (25), 7303-7307.

33. Chwastek, M.; Szumna, A., Higher Analogues of Resorcinarenes and Pyrogallolarenes: Bricks for Supramolecular Chemistry. Organic Letters 2020, 22, (17), 6838-6841.

34. Bourgeois, J.-M.; Stoeckli-Evans, H., Synthesis of New Resorcinarenes Under Alkaline Conditions. Helvetica Chimica Acta 2005, 88, (10), 2722-2730. 